\title{
Successful en bloc transplantation of a horseshoe kidney without division of the isthmus: first case reported in South Africa
}

\author{
A D Zarrabi, S G Wessels, L Vlok, A Van der Merwe \\ Department of Urology, University of Stellenbosch and Tygerberg Hospital \\ Corresponding author: Amir David Zarrabi (adz@sun.ac.za)
}

\begin{abstract}
Summary
This case is the first report of an en bloc transplantation of a horseshoe kidney in South Africa. The graft anatomy included complete fusion of the lower poles of the two kidney units with a single artery and vein associated with each unit. The thick isthmus of the graft kidney was supplied by a single, large caliber anomalous artery. Bench work (total duration 160 minutes) included the preparation of a short segment of donor aorta and IVC (containing the renal vessels) which were anastomosed to the recipient common iliac artery and common iliac vein respectively. The artery of the isthmus was anastomosed to the recipient right internal iliac artery. The graft was placed intraperitoneally. Cold ischaemia time was 15 hours, warm ischaemia time 144 minutes, total procedure time 450 minutes and estimated blood loss 1100 ml. Renogram ( ${ }^{99 m}$ Tc MAG-3) on days 1, 3 and 14 postoperatively revealed ATN. Hospital stay was 17 days and nadir creatinine at 6 weeks was $77 \mu \mathrm{mol} / 1$.
\end{abstract}

With a critical worldwide shortage of organs for transplantation, various strategies have been employed to increase the number of donor kidneys available. These include the use of kidneys from expanded criteria donors and non-heart beating donors. ${ }^{1}$ The growing discrepancy between the demand for kidneys and their availability further requires transplant surgeons to be able to utilize even select kidneys with congenital fusion abnormalities, provided that these organs would be likely to provide adequate and sustained renal replacement to the recipient.

Anatomical variations of the calyceal system, ureter, main vasculature and parenchyma are typically the rule when dealing with congenitally fused kidneys. Because of the multitude of possible anatomical configurations that the transplant surgeon may encounter, decision-making will be unique and dependent on the unique anomalies found in every case.

This report describes the successful transplantation of a horseshoe kidney from a brain-dead donor. Because of the presence of a substantially thick isthmus, the kidney masses were not divided and the recipient received both fused kidney portions en bloc. A thorough literature search was performed and yielded no previous reports of horseshoe kidney transplantation in South Africa (SA), though it would be reasonable to assume that transplantation of anatomically abnormal kidneys has previously been performed but not reported.

\section{Donor:}

A 38-year-old male was declared brain dead after suffering an intracerebral bleed secondary to hypertension. He had a normal serum creatinine level and no history of previous kidney problems or surgery.

\section{Recipient:}

Forty-seven-year-old female with end stage renal disease (ESRD) secondary to chronic glomerulonephritis (CGN). She had been on peritoneal dialysis (PD) for 2 years and was switched to haemodialysis (HD) 9 months before.

\section{Organ procurement:}

During organ harvesting a horseshoe kidney, ectopically located in the lower abdomen, was discovered. It was decided on-table that organ procurement would go ahead. Subsequently, a single, large, fused kidney was received by our transplant team. Generous segments of the donor aorta and inferior vena cava (IVC) were still intact and attached to all of the renal arteries and veins.

\section{Graft preparation:}

Extensive bench work (total duration 160 minutes) was required to precisely identify the unique anatomy of the graft: 


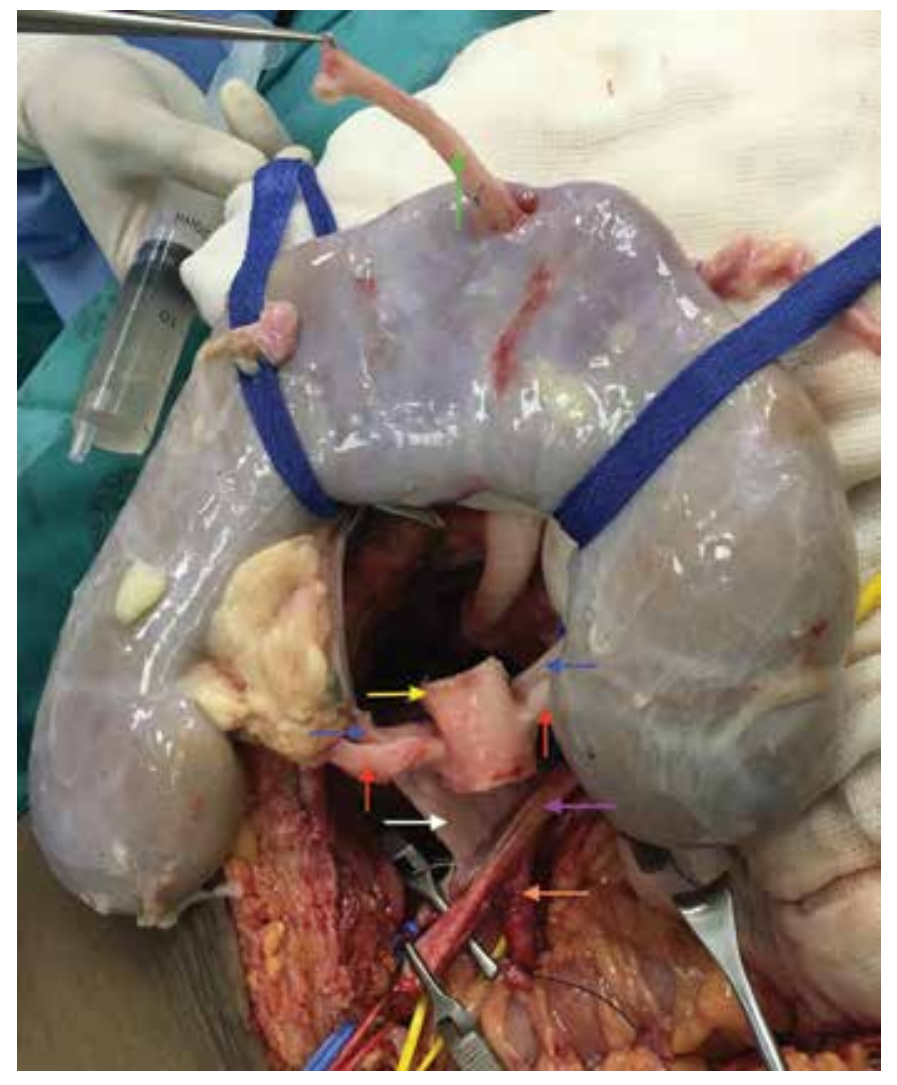

Fig. 1: Vascular anatomy of the horseshoe kidney and recipient vessels:

Red arrows: Graft renal arteries.

Yellow arrow: Graft aorta segment with proximal end sutured closed.

Blue arrows: Graft renal veins.

White arrow: Graft IVC.

Purple arrow: Recipient common iliac artery with arteriotomy. Orange arrow: Recipient internal iliac artery (tied and transected). Green arrow: Graft isthmus artery.

- Horseshoe kidney with complete fusion of the lower poles

- Substantially thick isthmus supplied by a single, large caliber artery

- Normal caliber ureter draining each renal pelvis

- A renal artery to each kidney, originating from contralateral sides of the aorta

- A renal vein draining each kidney, into contralateral sides of the IVC.

As part of the bench work a short segment of donor aorta was preserved, circumferentially intact, with ostia of the graft renal arteries on contralateral sides. The supra-renal end of the donor aorta was closed with a running Gorete ${ }^{\circledR}$ CV-6 non-absorbable suture and the other (distal) end left open for anastomosis to the recipient. A similar preparation of the donor IVC was performed with the supra-renal end closed and the proximal end left open for the anastomosis (Figure 1).

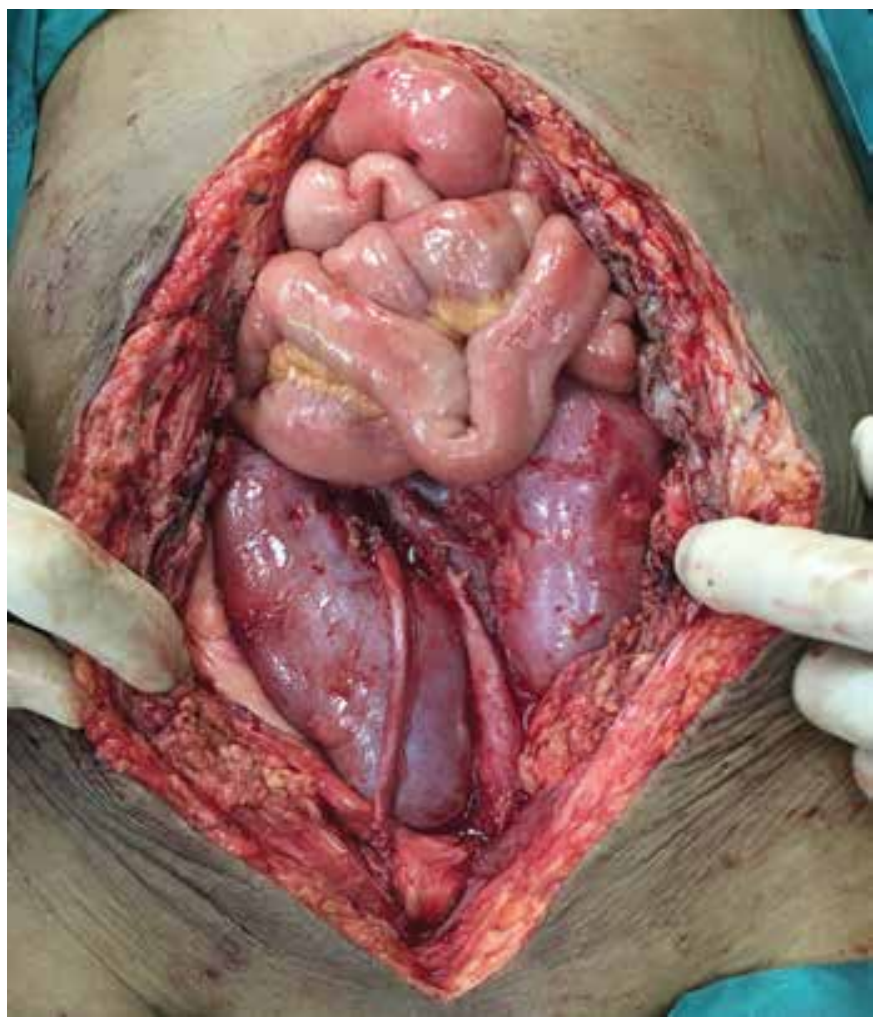

Fig. 2: Transplanted horseshoe kidney in intraperitoneal position (vascular clamps removed) with good perfusion of all areas.

\section{Graft implantation:}

Intraperitoneal graft implantation was performed via a midline laparotomy incision. The bowel was mobilized superiorly in order to gain access to the right common-, internal- and external iliac vessels. The vascular anastomoses (Figure 1) were performed as follows, using Goretex ${ }^{\circledR} \mathrm{CV}-6$ non-absorbable sutures:

- Graft renal arteries: End-to-side anastomosis of the open end of the graft aorta to the recipient common iliac artery.

- Graft renal veins: End-to-side anastomosis of the open end of the graft IVC to the recipient common iliac vein.

- Graft isthmus artery: End-to-end anastomosis to the recipient internal iliac artery.

Ischaemic times were as follows:

- Cold ischaemia: 15 hours

- Warm ischaemia: 144 minutes

- Venous anastomosis: 89 minutes

- Arterial anastomoses: 55 minutes.

Excellent perfusion was present immediately after removal of the vascular clamps (Figure 2). Bilateral ureteroneocystostomies were then performed (over JJ-stents) using a modified Lich-Gregoir approach on the extraperitoneal surface of the bladder in the space of Retzius.

The total procedure time was 450 minutes (including bench 
work of 160 minutes) and total estimated blood loss was $1100 \mathrm{ml}$. The relatively large volume of blood loss was related to the long duration of the procedure in a uraemic recipient as well as troublesome bleeding on release of the vascular clamps, which required a number of additional sutures at the anastomoses.

\section{Postoperative graft function:}

Initial delayed graft function was evident from low urine volumes and acute tubular necrosis (ATN) confirmed on ${ }^{99 \mathrm{~m}} \mathrm{Tc}$ MAG-3 renogram. Graft function gradually improved and the patient was discharged on day 17 postoperatively. Her nadir serum creatinine level at 6 weeks follow-up was $77 \mu \mathrm{mol} / \mathrm{l}$.

\section{Discussion}

The horseshoe kidney is probably the most common of all renal fusion abnormalities and occurs in about 1 in every 600 persons. $^{2}$ Typically, it behaves as a relatively harmless condition $(60 \%$ of patients remain asymptomatic) with a low requirement for operative intervention during the affected person's lifetime.

The experience with horseshoe kidney transplantation remains limited: Stroosma et al. published the findings of a worldwide survey in 2001 which indicated that on average only 3 horseshoe kidneys had been transplanted per year during the previous 25 years. ${ }^{3}$ Our own search of PubMed revealed reports of only 30 horseshoe kidney transplantations between 2001 and 2017, with the largest single-institution series consisting of 5 cases. When confronted with a horseshoe kidney for transplantation several strategies for implantation can be followed, depending on the specific anatomical variations found in each case. The following have been reported: 4,5

- Transplantation of the entire kidney mass en bloc into one recipient.

- Division of the isthmus and transplantation of both kidney portions into the same recipient.

- Division of the isthmus and transplantation of the two kidney portions into two different recipients.

- Division of the isthmus and discarding one kidney portion due to the presence of adverse factors (renal hypoplasia, severe vascular- or collecting system anomalies) and subsequent transplantation of the remaining portion into one recipient.
Division of the isthmus would typically be undertaken in cases where a thin, fibrotic isthmus were present. Division of a thick isthmus increases the risk of bleeding and urinary leak. ${ }^{6}$

A higher incidence of vesico-ureteric reflux (VUR), pelviureteric junction (PUJ) obstruction and ureteric duplication has been found in horseshoe kidneys and may predispose to urinary tract infections and urolithiasis. ${ }^{2}$ The presence of these abnormalities should be identified prior to transplantation of horseshoe kidneys as it may affect the quality of the graft.

Despite the high incidence of complex vascular anomalies, the incidence of postoperative vascular thrombosis does not seem to be higher than in transplantation involving normal kidneys. ${ }^{6}$

Though there is no data available from large series, reports indicate that the long-term outcomes for horseshoe kidney transplantation are similar to those of anatomically normal kidneys, provided that careful attention is paid to the unique anatomy of each case. ${ }^{7}$

This case confirms that transplantation of horseshoe kidneys can be safely undertaken in the South African transplant environment. In the rare event of being confronted with a horseshoe kidney for transplantation, careful consideration should be given to vascular, calyceal and ureteric anomalies as these may influence decision making.

\section{REFERENCES}

1. Bozkurt B, Kilic M. Marginal donors in renal transplantation. Transplant Proc. Jun 2015;47(5):1273-1281. doi: doi. org/10.1016/j.transproceed.2015.04.006. PMID: 26093697.

2. Bauer SB. Anomalies of the upper urinary tract. In: Wein AJ, Kavoussi LR, Novick AC, Partin AW, Peters CA. CampbellWalsh Urology. $9^{\text {th }}$ ed. Philadelphia: Elsevier, 2007:3287-91.

3. Stroosma OB, Schurink GW, Smits JM, Kootstra G. Transplanting horseshoe kidneys: a worldwide survey. J Urol. Dec 2001;166(6):2039-42. PMID: 11696702.

4. Guarrera JV, Arrington B, Birkhoff JD, et al. Back bench split of a deceased-donor horseshoe kidney for two transplant recipients. Kidney Int. Nov 2009;76(9):1012. doi: 10.1038/ ki.2009.250. PMID: 19829314.

5. Hau HM, Morgulk HM, Uhlmann D, et al. Horseshoe kidney for transplantation: technical considerations. Scand J Urol. Feb 2013;47(1):76-9. doi: 10.3109/00365599.2012.704940. PMID: 22835080 .

6. Nemes B, Kanyari Z, Zadori G, et al. Horseshoe kidney transplantation. Interv Med Appl Sci. Jun 2015;7(2):85-9. doi: 10.1556/1646.7.2015.2.7. PMID: 26120481.

7. Goyal A, Gaitonde K, Sagade SN, Shah BV, Kamat MH. Transplantation of horseshoe kidney from living-related donors: report of two cases. Transplant Proc. Feb 2003;35(1):32-4. PMID: 12591294. 